\title{
Self-reported health-related quality of life is an independent predictor of chemotherapy treatment benefit and toxicity in women with advanced breast cancer
}

\section{CK Lee*,1, MR Stockler ${ }^{1,2}$, AS Coates ${ }^{1,3}$, V Gebski', SJ Lord' and RJ Simes ${ }^{1,2}$ on behalf of Australian New Zealand Breast Cancer Trials Group}

'NHMRC Clinical Trials Centre, The University of Sydney, Camperdown, NSW, Australia; ${ }^{2}$ Sydney Cancer Centre - Royal Prince Alfred and Concord Hospitals, Sydney, NSW, Australia; '3nternational Breast Cancer Study Group, Bern, Switzerland

BACKGROUND: Baseline health-related quality of life $(\mathrm{QL})$ is associated with survival in advanced breast cancer. We sought to identify patients who were less likely to respond to chemotherapy and at greater risk of toxicity on the basis of their $\mathrm{QL}$.

METHODS: We used data from three advanced breast cancer trials in which patients $(n=378)$ were treated with cyclophosphamide, methotrexate and 5-fluouracil. Patients self-rated their QL using LASA scales for physical well-being (PWB), mood, pain, nausea/ vomiting, appetite and overall QL. Multivariable regression models were constructed to compare overall survival (OS), objective tumour response (OTR), adverse events (AEs) and weight loss according to grouped QL scores.

RESULTS: Physical well-being, mood, appetite and overall QL were significant univariable predictors of OS. Physical well-being and appetite remained significant after adjustment for baseline biomedical factors. Poor PWB was associated with lower OTR (odds ratio $(O R)=0.21$, 95\% confidence interval $(C l) 0.09-0.5 \mathrm{I})$, higher risk of non-haematological $A E s(O R=3.26,95 \% \mathrm{Cl} I .49-7.15)$ and greater risk of weight loss (OR 2.37, 95\% Cl I.12-5.0 I) compared with good PWB.

CONCLUSIONS: In women with advanced breast cancer, PWB and appetite are predictors of chemotherapy response and toxicity as well as survival. Quality of life should be a routine clinical assessment to guide patient selection for chemotherapy and for stratification of patients in clinical trials.

British Journal of Cancer (2010) I 02, I34I - 1347. doi:I0.1038/sj.bjc.6605649 www.bjcancer.com

Published online 13 April 2010

(C) 2010 Cancer Research UK

Keywords: quality of life; advanced breast cancer; treatment benefits; treatment toxicity

In advanced breast cancer, the primary goals of treatment are to prolong and improve quality of life (QL) because the disease is generally incurable. In a pivotal study conducted by Coates et al (1987), patient-rated QL improved significantly on average in women with advanced breast cancer receiving palliative chemotherapy, and both baseline QL scores and subsequent changes were prognostic for survival (Coates et al, 1992). Although chemotherapy is effective in relieving cancer-related symptoms, these average benefits may not accrue to all patients and may in some cases be offset by the significant physical and psychological side-effects. Therefore, appropriate selection of patients for chemotherapy is important to increase the likelihood that its benefits will likely outweigh its harms.

Determination of the prognosis of patients with advanced breast cancer can provide valuable information to guide oncologists in deciding whether to offer palliative chemotherapy. Oncologists consider a range of patient and disease characteristics such as age, performance status, disease-free interval, hormone receptor status, disease burden and prior adjuvant treatments (Hortobagyi et al, 1983; Gennari et al, 2005; Largillier et al, 2008). More recently,

*Correspondence: Dr CK Lee; E-mail: chee.lee@ctc.usyd.edu.au Received 23 December 2009; revised 13 March 2010; accepted 17 March 2010; published online 13 April 2010 patient's self-reported health-related QL has also been recognised as predictive of survival (Gotay et al, 2008; Quinten et al, 2009).

Poor QL before treatment is associated with shorter survival in patients with advanced breast cancer (Coates et al, 1992; Efficace et al, 2004; Winer et al, 2004; Gotay et al, 2008). However, there is only limited evidence of the relationship between baseline QL and response to chemotherapy (Seidman et al, 1995; Kramer et al, 2000; Winer et al, 2004) and treatment-related toxicity. Investigation of this issue is important because chemotherapy has a narrow therapeutic index and a fine balance between benefits and harms. Such information might help oncologists and patients to individualise their treatment decisions.

In this study, we used data from three trials conducted by the Australian New Zealand Breast Cancer Trials Group (ANZBCTG) to validate earlier findings that baseline QL predicts overall survival (OS) in advanced breast cancer and investigate the association between baseline QL and chemotherapy response and toxicity.

\section{MATERIALS AND METHODS}

We used data from the common control arms of three randomised controlled trials (RCTs) of first-line chemotherapy for patients with advanced breast cancer conducted by ANZBCTG (ANZ8101, ANZ8614 and ANZ0001). Trial participants were recruited from participating hospitals across Australia and New Zealand. 
ANZ8101, activated in June 1982, was a two-by-two factorial RCT comparing the efficacy of doxorubicin and cyclophosphamide $v s$ cyclophosphamide, methotrexate, 5-fluouracil and prednisone (CMFP), administered continuously $v s$ intermittently (Coates et al, 1987). ANZ8614, activated in January 1988, was a two-arm RCT comparing the efficacy of mitoxantrone $v s$ CMFP (Simes et al, 1994). ANZ0001, activated in June 2001, was a three-arm RCT comparing the efficacy of intermittent capecitabine $v s$ continuous capecitabine vs CMFP (Stockler et al, 2007).

All three trials included a measurement of patients' self-reported QL at baseline. Investigation of QL as a predictor of treatment response or toxicity was not specified in the original trial protocols.

\section{Patients}

Patients had histologically confirmed breast carcinoma with measurable or evaluable recurrent or metastatic disease; adequate bone marrow, hepatic and renal function; and were available for follow-up. Patients were excluded if they had received cytotoxic chemotherapy for recurrent or metastatic disease or extensive radiotherapy, or had a history of other cancer, diabetes mellitus or cardiac failure.

Only patients assigned to the continuous CMFP in each of the three trials were included in the present analysis. Patients assigned to the intermittent CMFP arm of ANZ8101 were excluded from this study because this treatment arm was inferior to the CMFP regimen given continuously as in the other trials. All patients provided written informed consent for participation in the trials.

\section{Treatments}

In each of the three trials, CMFP was administered in 28-day cycles with oral cyclophosphamide $\left(100 \mathrm{mg} \mathrm{m}^{-2}\right)$ daily for 14 days; intravenous methotrexate $\left(40 \mathrm{mg} \mathrm{m}^{-2}\right)$ and intravenous 5-fluorouracil $\left(600 \mathrm{mg} \mathrm{m}^{-2}\right)$ on days 1 and 8 . Oral prednisone $\left(40 \mathrm{mg} \mathrm{m}^{-2}\right)$ for first 14 days was routinely administered in patients from ANZ8101 and ANZ8614, and was optional in ANZ0001. All patients continued the initial chemotherapy regimen until disease progression, intolerance or unacceptable toxicity. Therapy beyond disease progression was at the discretion of the treating oncologist.

\section{QL instruments}

Patients self-reported their QL with five linear analogue self assessment (LASA) scales that measured physical well-being (PWB), mood, pain, nausea/vomiting and appetite (Priestman et al, 1977; Coates et al, 1993), and a single summative

Table I Demographic characteristics and chemotherapy treatment profile

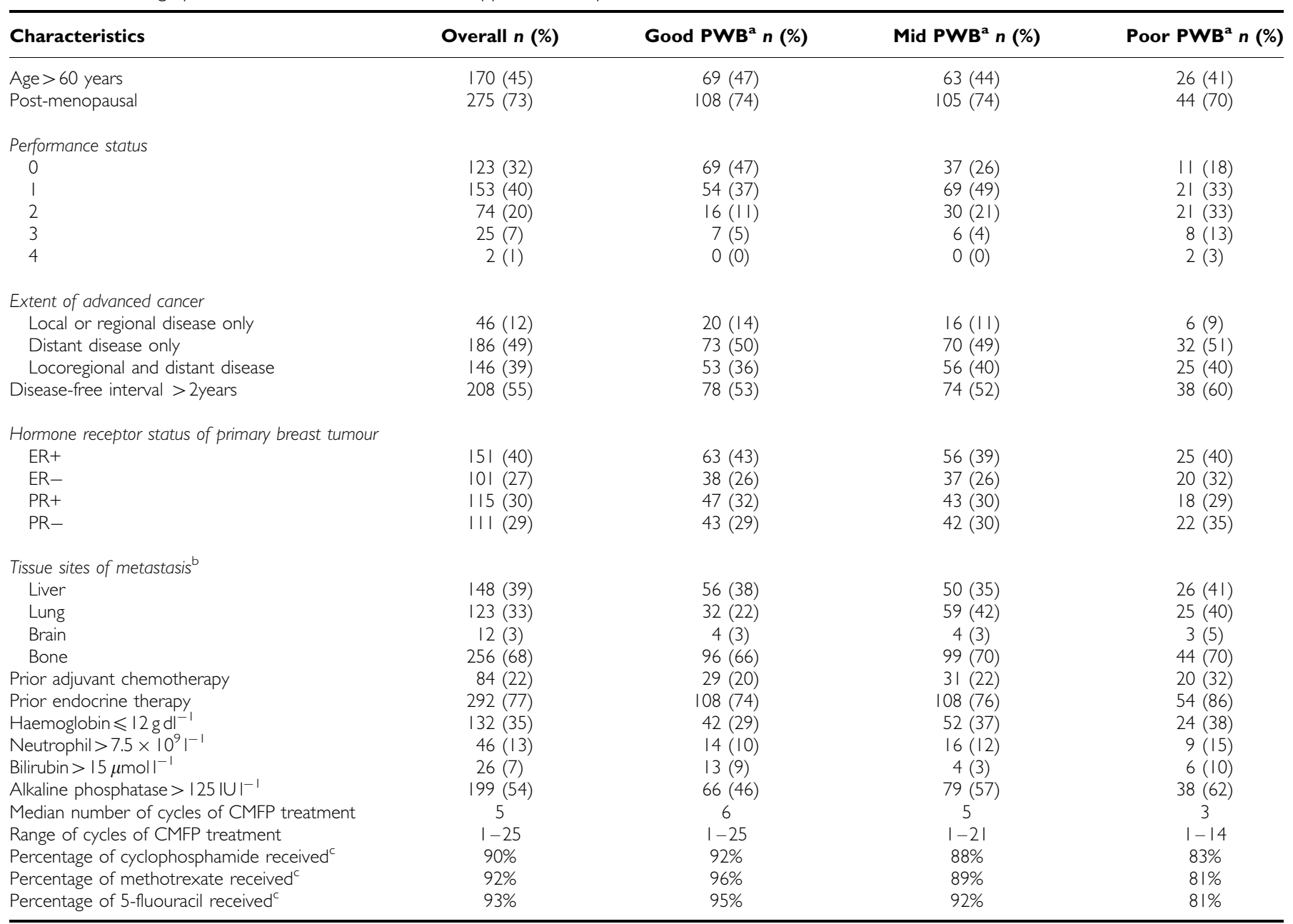

Abbreviations: CMFP = cyclophosphamide, methotrexate, 5-fluouracil, prednisone chemotherapy: $E R=$ oestrogen receptor; $P R=$ progesterone receptor: $P W B=$ physical wellbeing. ${ }^{a}$ Good PWB $=$ LASA score 0-25; mid PWB = LASA score 26-65; poor PWB = LASA score 66- 100. bMore than one site could have been involved, so percentages sum to more than $100 \%$. ${ }^{\top}$ The reported percentage is the median total dose of chemotherapy received as compared with the ideal dose calculated on the basis of the bodysurface area. 
LASA scale that measured overall QL (Spitzer et al, 1981). All scales were $100 \mathrm{~mm}$ long and scores range from 0 (best) to 100 (worst).

For the purpose of our analysis, scores for each QL scale were arbitrarily divided into three groups: good (0-25), mid $(26-65)$ or poor $(66-100)$. The categorisation was selected to be consistent with the cut-points used in a previous study (Coates et al, 1992).
Baseline OL, chemotherapy benefit and toxicity

CK Lee et al

\section{Treatment benefits}

Treatment benefits were evaluated by measuring OS, progressionfree survival (PFS), objective tumour response (OTR) and improvement in baseline body weight. OS and PFS were measured from randomisation to the date of death or first documented disease progression respectively. OTR rate was measured as the proportion of patients with evaluable disease

Table 2 Univariable Cox regression analysis of survival $(n=378)$

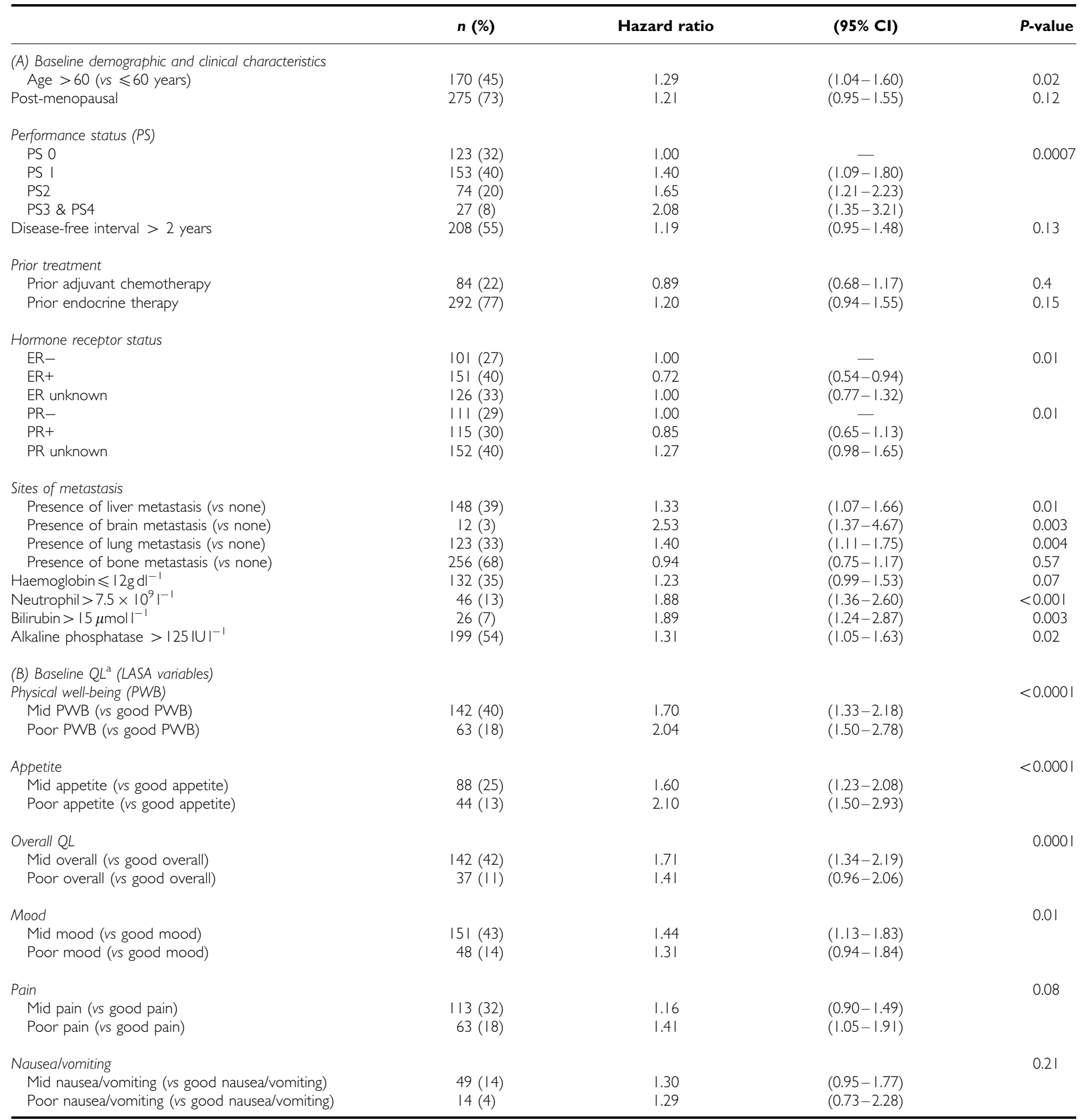

Abbreviations: $\mathrm{Cl}=$ confidence interval; $\mathrm{ER}=$ oestrogen receptor; $\mathrm{LASA}=$ linear analogue self-assessment; $\mathrm{PR}=$ progesterone receptor; $\mathrm{QL}=$ quality of life. ${ }^{\mathrm{a}} \mathrm{Good} \mathrm{QL}=\mathrm{LASA}$ score 0-25, Mid QL = LASA score 26-65, Poor QL = LASA score 66- 100. 
who achieved a complete response (CR) or partial response (PR). Weight loss ( $v s$ stable or weight gain) was measured as the proportion of patients with an average decrease in weight from their baseline reading.

\section{Treatment toxicity}

Adverse events (AEs) were expressed as the proportion of patients who developed any grade 3 or grade 4 toxicity during the first 4 cycles of chemotherapy treatment. World Health Organisation criteria (Miller et al, 1981) were used for ANZ8101 and ANZ8614; National Cancer Institute Common Toxicity Criteria (Arbuck et al, 1998) were used for ANZ0001. Haematological (anaemia, neutropenia and thrombocytopenia) and non-haematological (nausea, vomiting, diarrhoea, stomatitis and alopecia) AEs were analysed separately.

\section{Statistical analysis}

Overall survival and PFS were estimated by the Kaplan-Meier method, and differences between patients with good, mid and poor levels for each QL scale at baseline were compared with the logrank test (Kaplan and Meier, 1958). Cox proportional-hazard models were used to estimate differences in OS according to each level of the QL scale (Cox, 1972). Multivariable analysis for OS was first performed with backward stepwise selection of biomedical variables only. Then, multivariable analysis for OS was repeated with backward stepwise selection of biomedical and QL variables. Only statistically significant variables $(P<0.05)$ were retained in the final multivariable models. As shown in a previous analysis using the same data set (Lee et al, 2010), trial is a significant factor for survival; thus we stratified for trial in all univariable and multivariable analyses for this study. Formal tests to detect the presence of collinearity between the different QL scales, and QL scales and ECOG performance status were also performed (Weissfeld, 1989).

Univariable analyses with logistic regression tested for associations between QL subscales and OTR, AE and weight loss. Multivariable models for these outcomes were constructed to estimate the effects of these QL subscales, adjusted for biomedical variables found to be significant in Cox model for OS.

All analyses were two-sided with no adjustment for multiple comparisons. No imputation of missing baseline QL values was performed.

\section{RESULTS}

\section{Biomedical and demographic characteristics}

A total of 378 patients with a median follow-up of 4.8 years were included in this pooled analysis. The number of patients from ANZ8101, ANZ8614 and ANZ0001 were 75 (20\%), $194(51 \%)$ and $109(29 \%)$, respectively. The median follow-up of these patients from ANZ8101, ANZ8614 and ANZ0001 were 4.8 years (range $0-4.8$ years), 10.1 years (range $0-10.9$ years) and 3.2 years (range $0-4.8$ years), respectively. Table 1 summarises the baseline characteristics and the treatment profiles of these patients for the overall cohort and according to the baseline PWB. Apart from performance status and lung metastasis, distribution of baseline characteristics was similar in patients with good, mid and poor PWB. Similar patterns of baseline characteristics were observed for other QL scales (data not shown).

\section{Baseline QL scores}

Baseline LASA scores for each QL scale were available for $89-94 \%$ of the patients. The proportion of patients with self-rating for each scale was $93 \%$ (PWB), 94\% (mood), 93\% (pain), 93\% (nausea and vomiting), 93\% (appetite) and $89 \%$ (overall life quality). The distribution of the three categories of LASA scores for each QL subscale is shown in Table $2 \mathrm{~B}$.

\section{CMFP treatment}

Two patients randomised to CMFP chemotherapy in the original trials did not receive the assigned treatment. The remaining 376 patients $(99.5 \%)$ received a median five cycles of treatment. The chemotherapy doses administered were between 90 and $93 \%$ of those planned. Patients with good, mid and poor PWB received medians of 6, 5 and 3 cycles of CMFP, respectively (Table 1).

\section{OS and PFS}

Physical well-being, mood, appetite and overall QL were predictors of OS in the univariable analyses (Table 2B). Physical wellbeing and appetite were independent predictors of survival in a multivariable model with biomedical and QL variables (Table 3B). Performance status was statistically significant in a multivariate model with biomedical factors only (Table $3 \mathrm{~A}$ ), but was not statistically significant in a multivariable model with biomedical and QL scales (Table 3B). Patients with good PWB at baseline had a statistically significantly longer PFS and OS compared with patients who had mid or poor PWB (Figure 1A and $\mathrm{B})$. The median OS for patients with good, mid and poor $\mathrm{PWB}$ were 19, 11 and 9 months, respectively (log-rank $P<0.0001$ ). Similar results were observed for patients with good, mid and poor appetite (Figure 1C and D).

Significant collinearity was not detected between the different QL scales or between QL scales and ECOG performance status (results not shown).

Table 3 Multivariable Cox regression analyses of survival for demographic and baseline clinical characteristics, and for baseline demographic and clinical characteristics and QL variables $(n=378)$

\begin{tabular}{|c|c|c|c|}
\hline & Hazard ratio & $(95 \% \mathrm{Cl})$ & $P$-value \\
\hline \multicolumn{4}{|c|}{ (A) 'Best' model with demographic and clinical characteristics only } \\
\hline Age $>60$ & 1.46 & $(1.16-1.84)$ & 0.001 \\
\hline$E R-$ & 1.00 & - & 0.002 \\
\hline ER+ & 0.63 & $(0.47-0.84)$ & \\
\hline ER unknown & 0.94 & $(0.70-1.25)$ & \\
\hline Neutrophil $>7.5 \times 10^{9} 1^{-1}$ & 1.68 & $(1.18-2.40)$ & 0.004 \\
\hline PS 0 & 1.00 & - & 0.005 \\
\hline PSI & 1.44 & $(1.11-1.88)$ & \\
\hline PS2 & 1.60 & $(1.16-2.22)$ & \\
\hline PS3 \& PS4 & 1.83 & $(1.14-2.93)$ & \\
\hline Presence of brain metastasis (vs none) & 2.42 & $(1.28-4.57)$ & 0.006 \\
\hline Presence of liver metastasis (vs none) & 1.38 & $(1.08-1.77)$ & 0.009 \\
\hline Alkaline phosphatase $>125 \mathrm{IUI}^{-1}$ & 1.31 & $(1.03-1.67)$ & 0.03 \\
\hline \multicolumn{4}{|c|}{ (B) 'Best' model with demographic, clinical characteristics and QL variables } \\
\hline Neutrophil $>7.5 \times 10^{9} 1^{-1}$ & 1.91 & $(1.31-2.79)$ & 0.001 \\
\hline Age $>60$ & 1.45 & $(1.14-1.83)$ & 0.002 \\
\hline $\mathrm{ER}-$ & 1.00 & - & 0.004 \\
\hline ER+ & 0.64 & $(0.47-0.87)$ & \\
\hline ER unknown & 0.98 & $(0.72-1.33)$ & \\
\hline Good PWBa & 1.00 & - & 0.004 \\
\hline Mid PWB ${ }^{a}$ & 1.45 & $(1.09-1.91)$ & \\
\hline Poor PWB ${ }^{a}$ & 1.64 & $(1.12-2.40)$ & \\
\hline Presence of brain metastasis (vs none) & 2.37 & $(1.21-4.64)$ & 0.01 \\
\hline Alkaline phosphatase $>\left.|25| \mathrm{IU}\right|^{-1}$ & 1.37 & $(1.08-1.74)$ & 0.01 \\
\hline Good ppetite ${ }^{\mathrm{a}}$ & 1.00 & - & 0.03 \\
\hline Mid appetite & 1.36 & $(1.01-1.84)$ & \\
\hline Poor appetite ${ }^{a}$ & 1.49 & $(0.98-2.24)$ & \\
\hline
\end{tabular}

Abbreviations: $\mathrm{Cl}=$ confidence interval; $\mathrm{ER}=$ oestrogen receptor; $\mathrm{PWB}=$ physical well-being; PS = performance status; $\mathrm{QL}=$ quality of life; $U L N=$ upper limit normal.

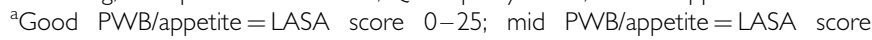
26-65; poor PWB/appetite $=$ LASA score 66- 100. 


\section{Objective tumour response}

The OTR rates for patients with good, mid and poor PWB were 47, 32 and $18 \%$, respectively (adjusted $P_{\text {trend }}<0.001$; Figure 2A). For patients with good, mid and poor appetite, the OTR rates were 41 , 34 and $18 \%$ respectively (adjusted $P_{\text {trend }}=0.02$; Figure $2 \mathrm{~A}$ ).

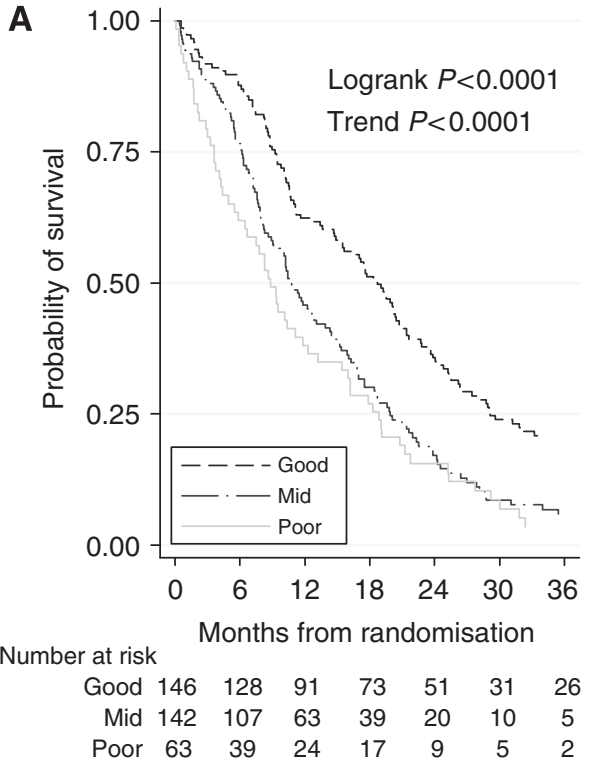

\begin{tabular}{lcc} 
PWB score & $\begin{array}{c}\text { Median overall survival } \\
\text { (months; 95\% Cl) }\end{array}$ & $\begin{array}{c}\text { Median progression-free survival } \\
\text { (months; 95\% Cl) }\end{array}$ \\
\hline Good (0-25) & $18.7(14.9-20.7)$ & $7.9(6.5-8.9)$ \\
Mid (26-65) & $10.8(8.9-13.9)$ & $5.5(4.7-7.1)$ \\
Poor (66-100) & $8.8(5.8-11.8)$ & $3.4(2.9-5.0)$ \\
\hline
\end{tabular}

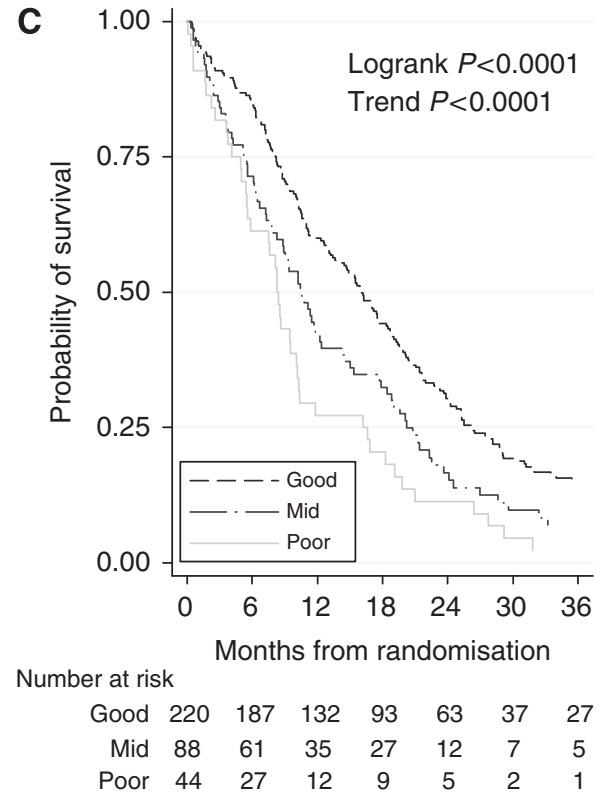

Appetite score Median overall survival (months; 95\% Cl)

\begin{tabular}{lc}
\hline Good (0-25) & $16.1(13.2-18.5)$ \\
Mid (26-65) & $10.6(8.0-12.4)$ \\
Poor $(66-100)$ & $8.3(5.5-10.1)$
\end{tabular}

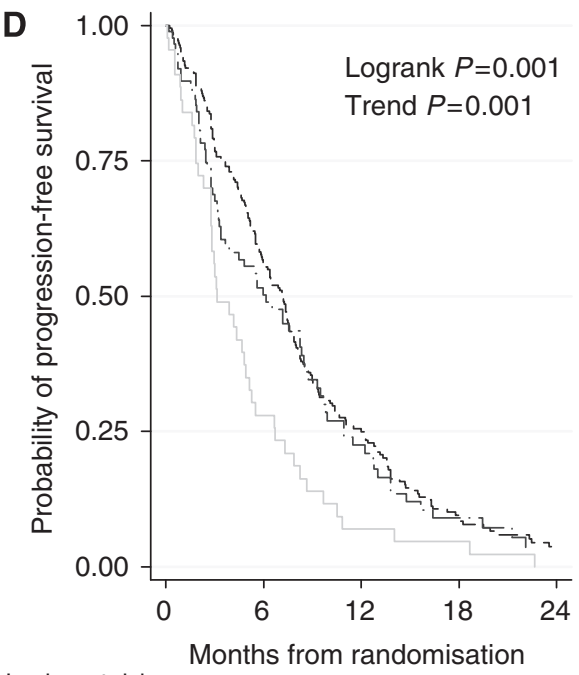

Number at risk

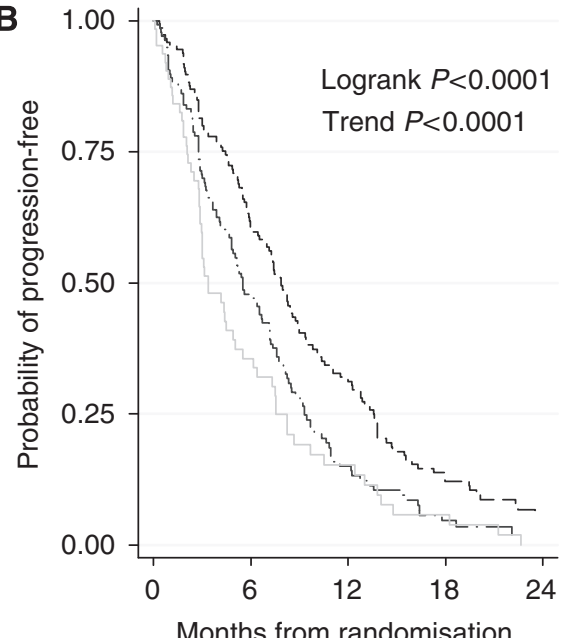

Number at risk

$\begin{array}{rcccc}\text { Good } 146 & 85 & 41 & 15 & 5 \\ \text { Mid } 142 & 61 & 17 & 5 & 1 \\ \text { Poor } 63 & 20 & 8 & 3 & 0\end{array}$

Median progression-free survival onths; $95 \% \mathrm{Cl}$

Fifty-one percent of women experienced weight loss during chemotherapy. Women with good PWB had a mean weight gain during chemotherapy of $2 \%$, but mean weight loss was $2 \%$ for women with mid PWB and $4 \%$ for women with poor PWB,

Weight loss
Median progression-free survival

$7.2(5.9-7.7)$

$6.2(3.4-8.4)$ $3.1(2.8-4.9)$ (months; 95\% Cl)

Figure I Overall survival $(\mathbf{A})$ and PFS (B) curves stratified by PWB score, and OS (C) and PFS (D) curves stratified by Appetite score. 

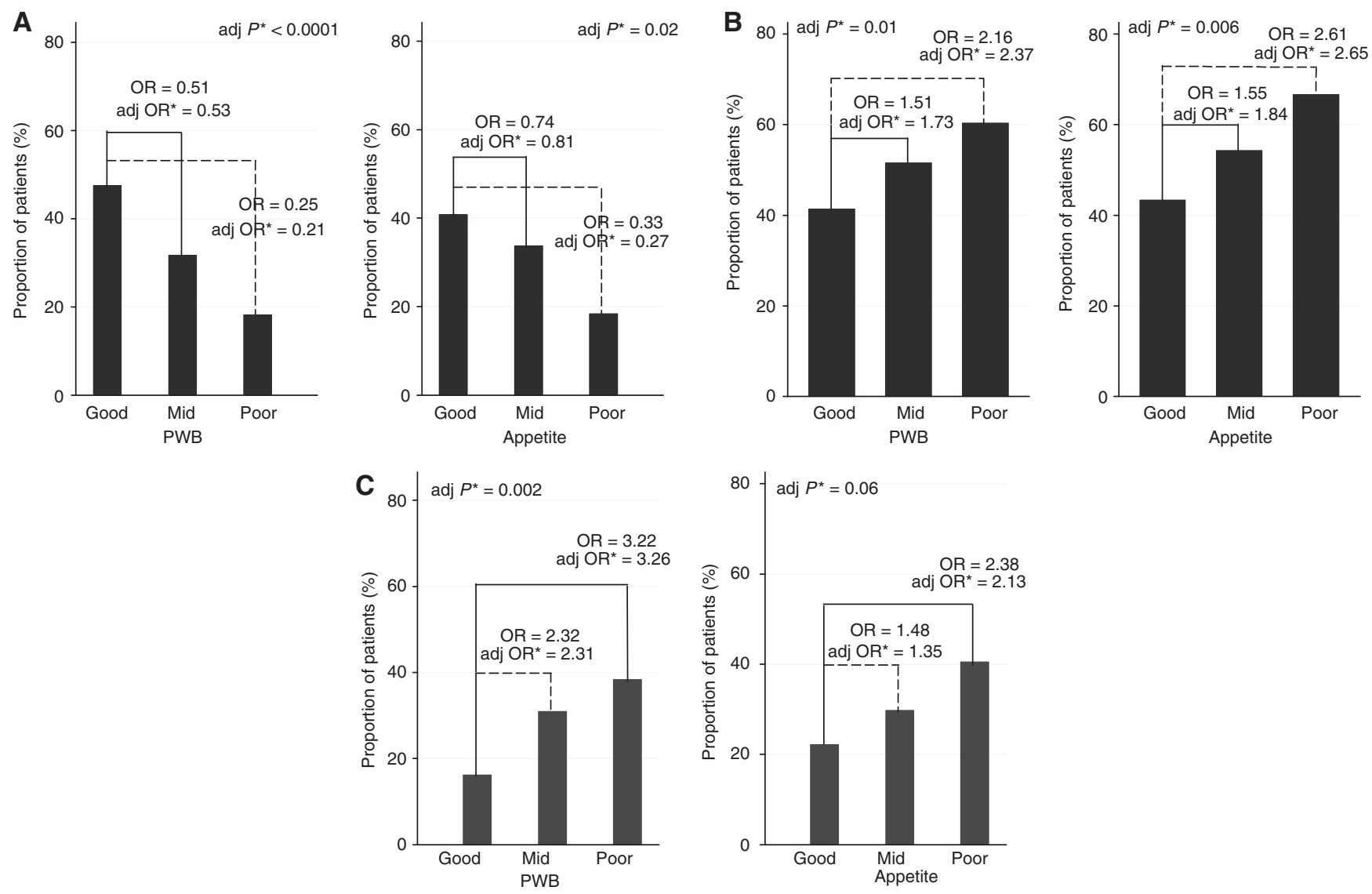

Figure 2 (A) Proportion of OTR stratified by PWB and Appetite scores. (B) Proportion with weight loss during chemotherapy stratified by PWB and Appetite scores. (C) Proportion with grade-3/4 non-haematological toxicity stratified by PWB and Appetite scores (*QL adjusted for performance status, age, liver, and brain metastasis, oestrogen receptor status, neutrophil, serum alkaline phosphatase and trial enrolment).

respectively (adjusted $P_{\text {trend }}=0.01$; Figure $2 \mathrm{~B}$ ). For women with good appetite, there was a mean weight gain of $0.5 \%$, but mean weight loss was $2 \%$ for women with mid appetite and $7 \%$ for women with poor appetite (adjusted $P_{\text {trend }}=0.006$; Figure $2 \mathrm{~B}$ ).

\section{Treatment toxicity}

Non-haematological AE rates were statistically significantly different for patients with good (16\%), mid (31\%) and poor $(38 \%)$ PWB (adjusted $P_{\text {trend }}=0.002$; Figure $2 \mathrm{C}$ ). Grade- 3 or 4 nausea and vomiting were reported in 19, 41 and $42 \%$; diarrhoea in 9, 12 and $11 \%$; and grade- 3 and 4 stomatitis in 8,11 and $17 \%$ of patients with good, mid and poor PWB, respectively. Grade- 3 and 4 alopecia rates were similar in the three PWB groups.

The rates of non-haematological AEs were not significantly different in patients with good, mid and poor appetite (adjusted $P_{\text {trend }}=0.06$; Figure $2 \mathrm{~B}$ ).

Grade- 3 and 4 haematological toxicity rates were not associated with PWB (adjusted $P_{\text {trend }}=0.92$ ) or appetite scores (adjusted $P_{\text {trend }}=0.60$ ).

\section{DISCUSSION}

In this analysis of women treated with CMFP as first-line chemotherapy for advanced breast cancer, patient self-reported PWB and appetite at baseline were independent predictors of OS and PFS. Women with poor PWB had a median OS that was 10 months shorter, and a median PFS that was 5 months shorter, than women with good PWB. Furthermore, women with poor PWB had OTR rates $29 \%$ lower, weight loss rates $19 \%$ lower and treatment-related non-haematological AE rates 22\% higher than women with good PWB. Similar findings were observed for women with poor appetite compared with those who had good appetite.

Our study also showed that women with poor QL derived less benefit and experienced more toxicity when treated with chemotherapy than women with good QL. Overall, women reporting poor QL at baseline received $50 \%$ fewer cycles of chemotherapy, were $20-30 \%$ less likely to benefit from chemotherapy and experienced rates of non-haematological treatment toxicity $20 \%$ higher than women reporting good QL at baseline.

This research adds to the growing evidence that QL is an independent predictor of survival in advanced cancer. In a systematic review of cancer trials, Gotay et al (2008) reported that 36 of 39 trials showed an association between QL and survival. Specific QL measures most frequently identified in these trials were overall QL, physical well being, appetite loss and pain. Another meta-analysis using data from 30 cancer trials produced a multivariable model that identified impaired physical functioning, pain and appetite loss as independent predictors of survival in addition to established biomedical factors (Quinten et al, 2009). Individual trials in advanced breast cancer have shown similar findings with PWB (Coates et al, 1992), appetite loss (Efficace et al, 2004), pain (Kramer et al, 2000) and overall QL (Seidman et al, 1995; Winer et al, 2004) all reported as independent predictors of survival. This study validates the prognostic significance of self-reported QL, and specifically of PWB and appetite, as predictors of survival in advanced breast cancer.

Furthermore, our findings raise the important question of whether measurement of baseline QL can be used to improve the selection of patients for chemotherapy. To date, few studies have investigated baseline QL as predictor of treatment benefit and/or 
toxicity in advanced breast cancer. Kramer et al (2000) reported an association between QL (dyspnoea, fatigue and overall QL) and tumour response in their analysis of 187 women treated with paclitaxel or doxorubicin, but selection of specific QL scales as independent predictors in the final multivariable model was reported as unstable because of multi-collinearity. They did not examine associations between QL and toxicity in this analysis. Two other studies reported no association between QL and tumour response to chemotherapy (Seidman et al, 1995; Winer et al, 2004) or treatment-related toxicity (Seidman et al, 1995). Possible explanations for the differences between these results and our study include small sample size (Seidman et al, 1995), the type of QL instruments and QL subscales used, and the methods used to assess tumour response.

The main strength of this study is the use of high-quality, individual patient data from three successive randomised clinical trials conducted by the same group. The pooled data set contains well-documented demographic, clinical and QL characteristics of trial participants who were treated with the same regimen of chemotherapy. Women in this study were assigned chemotherapy independent of their baseline QL. Follow-up and outcome data were collected prospectively with rigorous quality control.

This study has several limitations. First, our findings are from women with advanced breast cancer who were treated with CMFP. They may differ with other chemotherapy regimens or for other cancers (Quinten et al, 2009). Second, the results are not generalisable to women with early-stage breast cancer who do not have tumour-related symptoms (Coates et al, 2000; Goodwin et al, 2004; Gotay et al, 2008). Finally, this analysis was conducted post hoc using available trial data and therefore should be regarded as hypothesis-generating for future studies, rather than definitive.

If our findings are confirmed, trials of new treatment approaches should be investigated for women with poor QL. These trials could address the value of any chemotherapy $v s$ none, of less intense vs standard-intensity chemotherapy, or of chemotherapeutic or biological agents with a more favourable therapeutic-toxic ratio. Outcomes, which incorporate both survival time and QL, such as quality-adjusted PFS and OS, may be more relevant measures of treatment benefit in these women. The results of this study also suggest the value of stratifying patients by their baseline QL for future randomised trials in advanced breast cancer.

The primary goal of chemotherapy in advanced breast cancer is to prolong and improve QL. Our findings suggest that women with poor QL derive less benefit from chemotherapy and have increased risks of toxicity than women with good QL. This analysis should be regarded as hypothesis-generating and future studies that examine the role of chemotherapy in advanced breast cancer patients with poor QL are warranted.

\section{REFERENCES}

Arbuck S, Ivy S, Setser A (1998) The Revised Common Toxicity Criteria: Version 2.0: CTEP Website. http://ctep.info.nih.gov

Coates A, Gebski V, Bishop J, Jeal P, Woods R, Snyder R, Tattersall M, Byrne M, Harvey V, Gill G (1987) Improving the quality of life during chemotherapy for advanced breast cancer. A comparison of intermittent and continuous treatment strategies. N Engl J Med 317: 1490-1495

Coates A, Gebski V, Signorini D, Murray P, McNeil D, Bryne M, Forbes J (1992) Prognostic value of quality-of-life scores during chemotherapy for advanced breast cancer. J Clin Oncol 10: 1833-1838

Coates A, Thomson D, McLeod G, Hersey P, Gill PG, Olver IN, Kefford R, Lowenthal RM, Beadle G, Walpole E (1993) Prognostic value of quality of life scores in a trial of chemotherapy with or without interferon in patients with metastatic malignant melanoma. Eur J Cancer 29A: 1731-1734

Coates AS, Hurny C, Peterson HF, Bernhard J, Castiglione-Gertsch M, Gelber RD, Goldhirsch A (2000) Quality-of-life scores predict outcome in metastatic but not early breast cancer. J Clin Oncol 18: 3768-3774

Cox DR (1972) Regression models and life-tables. J Royal Stat Soc 34: $187-220$

Efficace F, Biganzoli L, Piccart M, Coens C, Van Steen K, Cufer T, Coleman RE, Calvert HA, Gamucci T, Twelves C, Fargeot P, Bottomley A (2004) Baseline health-related quality-of-life data as prognostic factors in a phase III multicentre study of women with metastatic breast cancer. Eur J Cancer 40: $1021-1030$

Gennari A, Conte P, Rosso R, Orlandini C, Bruzzi P (2005) Survival of metastatic breast carcinoma patients over a 20 -year period. Cancer 104: $1742-1750$

Goodwin PJ, Ennis M, Bordeleau LJ, Pritchard KI, Trudeau ME, Koo J, Hood N (2004) Health-related quality of life and psychosocial status in breast cancer prognosis: analysis of multiple variables. J Clin Oncol 22: $4184-4192$

Gotay CC, Kawamoto CT, Bottomley A, Efficace F (2008) The prognostic significance of patient-reported outcomes in cancer clinical trials. J Clin Oncol 26: $1355-1363$

Hortobagyi G, Smith T, Legha S, Swenerton K, Gehan E, Yap H, Buzdar A, Blumenschein G (1983) Multivariate analysis of prognostic factors in metastatic breast cancer. J Clin Oncol 1: 776-786

Kaplan EL, Meier P (1958) Nonparametric estimation from incomplete observations. J Am Stat Assoc 53: 457-481

Kramer JA, Curran D, Piccart M, de Haes JCJM, Bruning P, Klijn J, Van Hoorebeeck I, Paridaens R (2000) Identification and interpretation of clinical and quality of life prognostic factors for survival and response to treatment in first-line chemotherapy in advanced breast cancer. Eur J Cancer 36: $1498-1506$
Largillier R, Ferrero J-M, Doyen J, Barriere J, Namer M, Mari V, Courdi A, Hannoun-Levi JM, Ettore F, Birtwisle-Peyrottes I, Balu-Maestro C, Marcy PY, Raoust I, Lallement M, Chamorey E (2008) Prognostic factors in 1038 women with metastatic breast cancer. Ann Oncol 19: 2012-2019

Lee CK, Lord SJ, Stockler MR, Coates AS, Gebski V, Simes RJ (2010) Historical cross-trial comparisons for competing treatments in advanced breast cancer - an empirical analysis of bias. Eur J Cancer 46: 541 - 548

Miller AB, Hoogstraten B, Staquet M, Winkler A (1981) Reporting results of cancer treatment. Cancer 47: 207-214

Priestman T, Baum M, Jones V, Forbes J (1977) Comparative trial of endocrine versus cytotoxic treatment in advanced breast cancer. $\mathrm{Br} \mathrm{Med}$ $J$ 1: $1248-1250$

Quinten C, Coens C, Mauer M, Comte S, Sprangers MAG, Cleeland C, Osoba D, Bjordal K, Bottomley A (2009) Baseline quality of life as a prognostic indicator of survival: a meta-analysis of individual patient data from EORTC clinical trials. Lancet Oncol 10: 865-871

Seidman AD, Portenoy R, Yao T-J, Lepore J, Mont EK, Kortmansky J, Onetto N, Ren L, Grechko J, Beltangady M, Usakewicz J, Southrada M, Houston C, McCabe M, Salvaggio R, Thaler H, Norton L (1995) Quality of life in phase II trials: a study of methodology and predictive value in patients with advanced breast cancer treated with paclitaxel plus granulocyte colony-stimulating factor. J Natl Cancer Inst 87: 1316- 1322

Simes R, Gebski V, Coates A, Forbes J, Harvey V, Van Hazel G, Tattersall M, Abdi E, Brigham B (1994) Quality of life with single agent mitozantrone or combination chemotherapy for advanced breast cancer, a randomised trial. Proc Am Soc Clin Oncol 13: 73

Spitzer W, Dobson A, Hall J, Chesterman E, Levi J, Shepherd R, Battista RN, Catchlove BR (1981) Measuring the quality of life of cancer patients: a concise QL index for use by physicians. J Chronic Dis 34: 585-597

Stockler M, Sourjina T, Harvey V, Frances P, Byrne M, van Hazel G, Fitzharris B, Ackland S, Finch K, Lindsay D, Kato-Fong A, Paksec L, Gebski V, Simes R, Coates A, Forbes J (2007) A randomized trial of capecitabine given intermittently versus continuously versus classical CMF as first line chemotherapy for women with advanced breast cancer unsuited to more intensive treatment. Breast Cancer Res Treat 100: S278

Weissfeld LA (1989) A multicollinearity diagnostic for models fit to censored data. Commun Stat Theory Methods 18: 2073-2085

Winer EP, Berry DA, Woolf S, Duggan D, Kornblith A, Harris LN, Michaelson RA, Kirshner JA, Fleming GF, Perry MC, Graham ML, Sharp SA, Keresztes R, Henderson IC, Hudis C, Muss H, Norton L (2004) Failure of higher-dose paclitaxel to improve outcome in patients with metastatic breast cancer: cancer and leukemia group B trial 9342. J Clin Oncol 22: $2061-2068$ 